\title{
Glucocorticoid Induced Leucine Zipper in Lipopolysaccharide Induced Neuroinflammation
}

\author{
Emily Witek ${ }^{1}$, Debra Hickman ${ }^{1}$, Debomoy K. Lahiri ${ }^{2 \star}$ and Mythily Srinivasan ${ }^{3 *}$ \\ ${ }^{1}$ Laboratory Animal Resources and Center, Department of Internal Medicine, Indiana University School of Medicine, \\ Indianapolis, IN, United States, ${ }^{2}$ Department of Psychiatry, Stark Neuroscience Research Institute, Indiana University School \\ of Medicine, Indianapolis, IN, United States, ${ }^{3}$ Department of Oral Pathology, Medicine and Radiology, Indiana University \\ School of Dentistry and Indiana University - Purdue University Indianapolis, Indiana University School of Medicine, \\ Indianapolis, IN, United States
}

Glucocorticoids (GCs) are steroid hormones secreted as the end-product of the neuroendocrine stress cascade. Both absence and elevated GC mediate neurotoxic responses, suggesting that a narrow window ranging from physiological to slightly high GC mediate protective responses. The beneficial effects of GC are attributed to the transactivation of regulatory proteins and inhibition mediated by glucocorticoid receptor (GR) interactions with other co-factors. The glucocorticoid induced leucine zipper (GILZ) is a gene strongly upregulated by GC and mediates many of the anti-inflammatory and anti-proliferative effects of GC. Although GILZ is constitutively expressed in many tissues including the brain, the expression has been shown to occur with varying dynamics suggesting that the local milieu modulates its expression with consequent

OPEN ACCESS

Edited by:

Merce Pallas,

University of Barcelona, Spain

Reviewed by:

Cristoforo Scavone,

University of São Paulo, Brazil Kaoru Tominaga,

Jichi Medical University, Japan

*Correspondence:

Debomoy K. Lahiri dlahiri@iupui.edu

Mythily Srinivasan

mysriniv@iupui.edu

Received: 28 August 2018 Accepted: 17 December 2018 Published: 25 January 2019

Citation:

Witek E, Hickman D, Lahiri DK and Srinivasan M (2019) Glucocorticoid Induced Leucine Zipper in Lipopolysaccharide Induced Neuroinflammation.

Front. Aging Neurosci. 10:432. doi: 10.3389/fnagi.2018.00432 effects on cellular responses. Here we investigated the expression profile of GILZ in lipopolysaccharide (LPS) mediated neuroinflammation model of Alzheimer's disease (AD). Our data suggest that the GILZ expression is downregulated in neuroinflammation correlating inversely with the pro-inflammatory cytokines and innate immune responses.

Keywords: neuroinflammation, glucocorticoid induced leucine zipper, Alzheimer's disease, toll like receptor-4, cytokines

\section{INTRODUCTION}

Increased plasma glucocorticoids (GCs) are features of both aging and Alzheimer's disease (AD; Huang et al., 2009). Endogenous GC released by the adrenal glands play a key role in the homeostasis by regulating energy metabolism, coordinating immune responses and orchestrating the adaptive responses to stress. Increased secretion of GC can occur in response to various stimuli such as low plasma levels of GC, psychological or physical stress. The elevated GC in general is suppressed by a negative feedback loop, mainly regulated by the hypothalamic pituitary adrenal axis (HPA; Abrahám et al., 2006; Vyas et al., 2016). However, aging has been associated with a decreased sensitivity of the HPA axis to GC feedback, which in turn leads to prolonged elevated levels of plasma GC (Lupien et al., 1999).

Although the profound anti-inflammatory effects of GC suggest a neuroprotective role, emerging evidence strongly implicate elevated GC in potentiating the pro-inflammatory responses and neuroinflammation in the central nervous system (CNS). Multiple mechanisms are suggested for the effects of high GC on brain including dysregulated HPA axis, alterations in glial functions as well as structural remodeling of neurons with synaptic loss 
(Duque Ede and Munhoz, 2016; Vyas et al., 2016). Classically, GC act by binding the glucocorticoid receptor (GR) or the mineralocorticoid receptor (MR) in the cytoplasm. The ligand bound GR complex translocate to the nucleus and mediate cellular responses by genomic mechanisms via specific glucocorticoid response elements (GREs) in the DNA or by non-genomic protein: protein interactions. The therapeutic efficacy of GC are attributed to the combined effects of transactivation of regulatory proteins and inhibition of the pro-inflammatory transcription factor, the nuclear factorkappa B (NF-кB) by tethering (Newton, 2000; Srinivasan and Lahiri, 2017).

Glucocorticoid induced leucine zipper (GILZ) is a member of the TSC22 family of proteins that share the TSC box and the leucine zipper domains. It was originally identified as a gene rapidly induced by dexamethasone in lymphocytes (D'Adamio et al., 1997). More recently, its constitutive expression is reported in multiple cell types such as the microglia, epithelial cells, skeletal muscle cells, osteocytes and cardiomyocytes (Shi et al., 2003; Yachi et al., 2007; Liu et al., 2013; Pan et al., 2014). Functionally, GILZ mimics the anti-inflammatory and the anti-proliferative effects of GC by inhibiting the activated NF- $\kappa$ B and by upregulating the transcription of anti-inflammatory genes such as IL-10 (Berrebi et al., 2003; Mazzon et al., 2014).

GILZ is induced in the brain by both MR and GR mediated transactivation (Bergann et al., 2011). Since GC exhibit over 10-fold higher affinity for MR than GR, it is likely that under physiological conditions of low GC, GILZ induced by the high affinity MR assists in maintaining the homeostasis in the CNS. When GC is elevated, impaired feedback and increased cytoplasmic retention of GC could result in reduced GILZ induction contributing to dysregulated cellular responses (Rupprecht et al., 1993; Ogita et al., 2012). Stress induced GC upregulates the toll like receptors (TLRs) in microglia primed by immune activation (Frank et al., 2010). In addition, GILZ induction has been shown to occur with varying dynamics in different tissues in vivo, suggesting that the local environment can modulate its expression with potential effects on cellular responses (Ayyar et al., 2015).

In this study, we investigated the role of GILZ in lipopolysaccharide (LPS) induced neuro-inflammation and its relation to the innate immune response in the brain. LPS induced inflammation is a widely used model to study the mechanisms of neuroinflammation preceding neurodegeneration in $\mathrm{AD}$ pathogenesis (Nazem et al., 2015). Our data suggest that the GILZ is downregulated and inversely related to the increased TLR-4 expression in the brain in LPS induced neuroinflammation.

\section{MATERIALS AND METHODS}

\section{Animals and Disease Induction}

LPS from Escherichia coli 026:B6, = 10,000 EU/mg, was purchased from Sigma (Sigma Aldrich, St. Louis, MO, USA). Groups of adult C57/6J mice (five/group) were administered intraperitoneally either LPS at $250 \mathrm{mg} / \mathrm{kg}$ in $100 \mu \mathrm{l}$ of sterile saline followed by corn oil daily for 6 days (Ifuku et al., 2012;
Catorce and Gevorkian, 2016) or only saline (100 $\mu \mathrm{l})$ injections. Un-manipulated naïve mice were included as additional controls. The animal experiments were approved by the Institutional animal care and use committee of the Indiana University Purdue University at Indianapolis.

\section{Histology and Immunohistochemistry}

The mice were sacrificed on day 7 by intra-cardial perfusion of $25 \mathrm{ml}$ of $0.9 \%$ saline. Blood was collected prior to infusion. Brain from each mouse was removed, one half was immediately frozen and the other half fixed in $4 \%$ paraformaldehyde for paraffin embedding. Hematoxylin and eosin stained sections were assessed for inflammation. Serial $10 \mu$ thick coronal sections were immunostained for markers of microglial cells and astrocytes, GILZ and NF- $\kappa$ B p65 (Ray et al., 2009). Briefly, after deparaffinization, hydration, antigen retrieval and blocking non-specific binding (Enzo biosciences, Farmingdale, NY, USA) the sections were incubated overnight with the anti-Iba-1 (Clone:AIF1) or anti-glial fibrillary acidic protein (GFAP; clone GA5) or anti-NFKB p65 (clone 12H11; EMD Millipore Corporation, Temecula, CA, USA) or anti-GILZ polyclonal antibody (Cat \#PA5-34506; Invitrogen, Carlsbad, CA, USA) primary antibody. Brown staining observed by using the IHC select peroxidase conjugated streptavidin-biotin system was considered positive (Millipore). For each marker, the specificity of staining was confirmed by incubating a separate set of sections with secondary antibodies alone. The area of positive staining in the dentate gyrus (DG) and the Cornu Ammonis (CA1 and CA3) regions of each stained section was quantified by ImageJ software (NIH Image 1.62).

\section{Enzyme Linked Immunosorbent Assay}

The cytokines IL- 6 , TNF- $\alpha$, and IL-17 in serum from each blood sample was quantitated using OptEIA kits (BD Biosciences, San Jose, CA, USA).

\section{Real Time Polymerase Chain Reaction}

Total cellular RNA isolated from each brain tissue using Qiagen kit (Invitrogen, Carlsbad, CA, USA) was reverse transcribed using iScript cDNA kit (Biorad, Hercules, CA, USA). Equal amount of CDNA was used for amplification of $\beta$-actin, IL-1 $\beta$, IL-12, CD14, TLR-4 and CD4 by quantitative real time polymerase chain reaction (PCR) using SYBR green/ROX qPCR master mix (SA Biosciences, Frederick, MD, USA) on the ABI Prism 7000 sequence detection system (Applied Biosystem, Foster City, CA, USA; Srinivasan and Janardhanam, 2011). The primers (gene accession number) used were: $\beta$-actin (NM_007393): F-5'TC ATGAAGTGTGACGTTGACATCCGTA3'; R-5'CCTAGAAG CATTTGCGCTGCACGATGG3' (286bp); IL1ß (NM_008361) F-5'AGCTGATGGCCCTAAACAGA3'; R-5'GGTCGGAGATT CGTAGCTGG3' (89bp); CD14 (NM_009841) F-5'GAGCTAG ACGAGGAAAGTTGT3'; R-5'ACCGTAAGCCGCTTTAAGGA CAGA3' (206bp); GILZ (NM_001077364) F-5'CTAGCTCCG CAGGTGCGCAC3'; R-5'CGAGGCCAACAGGTGAGCGG3' (122bp), IL-12 (NM_001303244) F-5'GGAAGCACGGCAGCA 
GAATA3'; R-5'AACTTGAGGGAGAAGTAGGAATGG3' (179bp), TLR4 (NM_021297) F-5'CAGTCGGTCAGCAAACGC CTTCTTC; R-5'TGTAACTGGTGGCAGCGCA3' (216bp); CD4 (NM_013488) F-5'GAGAGTCAGCGGAGTTCTC3'; R-5'CTCACAGGTCAAAGTATTGTTG3' (182bp). The gene specific threshold cycle $(\mathrm{Ct})$ was corrected by subtracting the $\mathrm{Ct}$ for the housekeeping gene $\beta$-actin. The magnitude of change in each gene was determined by the $2^{-\Delta \mathrm{Ct}}$ method. Each measurement of a sample was performed in duplicates and the experiments were repeated at least two times.

\section{Statistical Analysis}

Pairwise Student's $t$-test was used to determine statistical significance in the cytokine and mRNA expression between the naïve, saline and LPS treated groups. $p<0.05$ was considered significant. The relationship between the GILZ and TLR4 transcripts was evaluated by regression analysis, Pearson and Spearman correlation coefficient.

\section{RESULTS}

\section{LPS Induced Neuroinflammation Exhibits Increased Gliosis and Reduced GILZ Protein Expression}

Peripherally administered LPS has been shown to increase the number of activated microglial cells as well as astrogliosis in the brain (Qin et al., 2007; Catorce and Gevorkian, 2016). We observed that the mean area of Iba+ microglial (Figures 1B,I) and GFAP+ astrocytes staining (Figures 1D,I) was higher in specific regions of the hippocampus of mice subjected to LPS induced neuroinflammation as compared to that in vehicle treated mice (Figures 1A,C,I). We also observed that the p65 immununostaining was higher in the DG and CA1 regions of the hippocampus of LPS administered mice (Figures 1F,I) as compared to that of the control mice (Figures 1E,I). While the control group of mice exhibited increased GILZ staining (Figures 1G,I), GILZ+ cells were fewer in DG and CA1 region the hippocampus of mice subjected to LPS induced neuroinflammation (Figures 1H,I).

\section{The GILZ Transcript Correlates Inversely With the Innate Immune and Inflammatory Transcripts in LPS Induced Neuroinflammation}

The inflammatory cytokines TNF- $\alpha$ and IL-17 was significantly higher in the serum of mice subjected to LPS induced neuroinflammation as compared to the serum of control group (Figure 2B). TLR4 is one of the main LPS recognizing receptors that respond to inflammatory stimuli and mediate NF- $\kappa B$ signaling pathway in microglial cells (Yu et al., 2015; Lykhmus et al., 2016). We observed that the expression of the TLR4 mRNA (Figures 2A,G) and that of its co-receptor CD14 (Figures 2A,H) was upregulated in the brain tissues of mice subjected to LPS induced neuroinflammation as compared to that in control mice. The upregulation was also observed for the transcripts

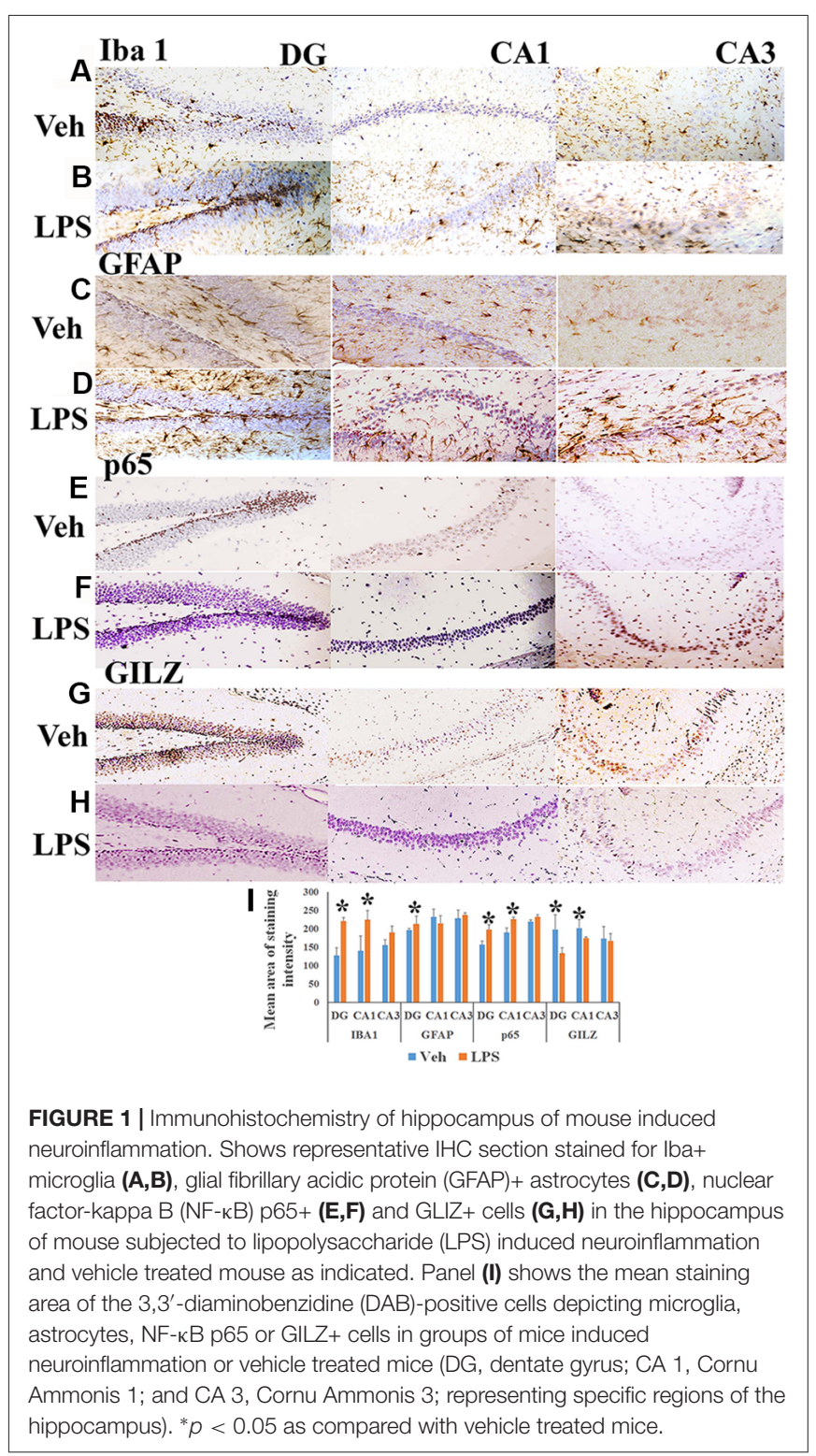

of inflammatory markers CD4 and IL-1 $\alpha$ (Figures 2A,C-E). In contrast, the GILZ mRNA was significantly lower in the brain tissues of mice subjected to LPS induced neuroinflammation as compared to that of the control group (Figures 2A,F). Regression analysis suggests a strong positive correlation between GILZ and TLR-4 (Spearman $R=0.9$ ) expression in individual mouse in the control group (Figure 2I). This relationship was lost in individual mice subjected to LPS induced neuro-inflammation (Figure 2J).

\section{DISCUSSION}

Brain is well recognized as a major neural target for the actions of GC. The paradoxical effects of GC in neuronal survival and death have been attributed to the concentration and duration of exposure of GC and the bioavailability of receptors. In physiological conditions and during circadian trough, GC 


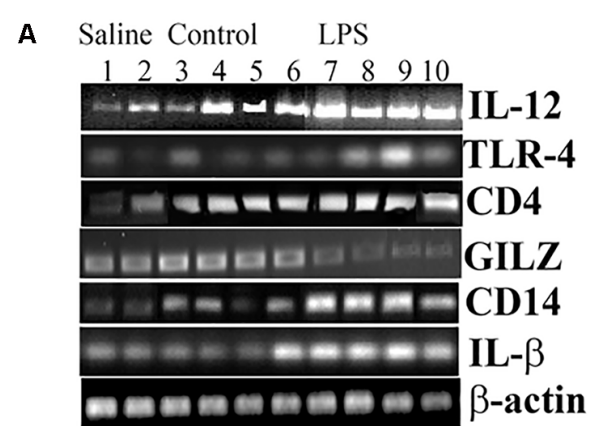

C

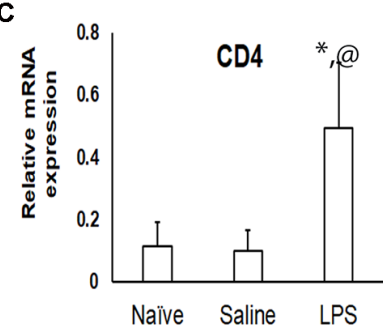

D

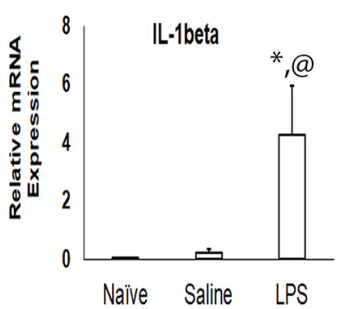

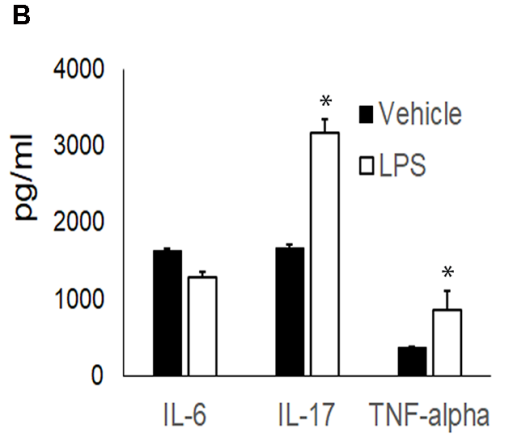

$\mathbf{E}$
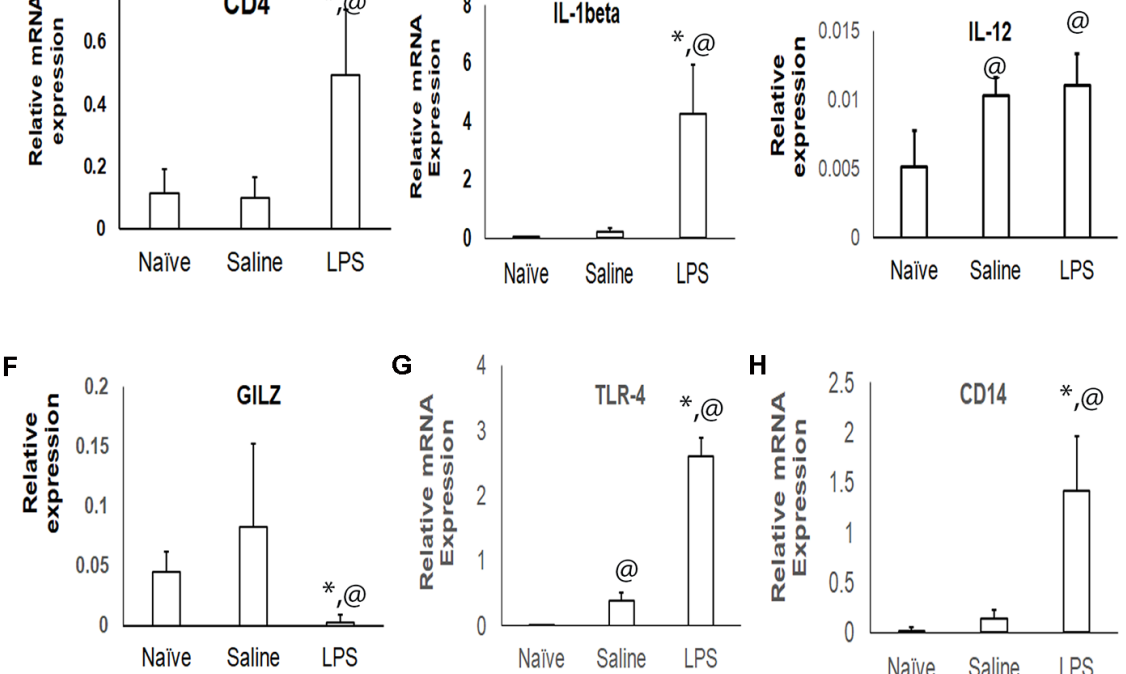

I
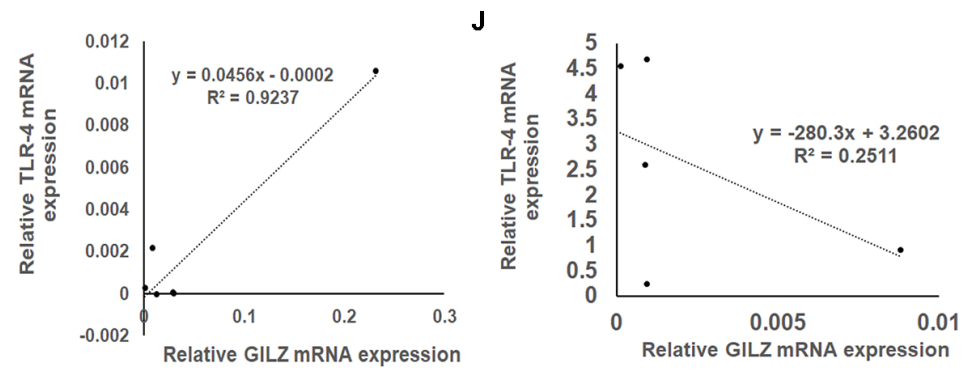

FIGURE 2 | Effect of LPS induced neuroinflammation in the expression profiles of inflammatory markers and GILZ: groups of adult C57BL/6 mice were subjected to LPS induced neuroinflammation as described in the "Materials and Methods" section. Equal quantity of cDNA isolated from brain tissues were amplified for IL-1 $\beta$, CD14, toll like receptor-4 (TLR-4), IL-12, CD4 and $\beta$-actin mRNA by quantitative real time polymerase chain reaction (PCR). (A) Gel electrophoresis of the PCR products $\beta$-actin IL-1 $\beta$, CD14, TLR-4, GILZ, IL-12 and CD4. Panel (B) shows indicated inflammatory cytokines in the circulation as determined by enzyme linked immunosorbent assay (ELISA). (C-H) Relative mRNA quantitation of the indicated product with respect to that of housekeeping gene $\beta$-actin is shown. Data are average $\pm \mathrm{SD} .{ }^{*}$ and ${ }^{@} p<0.05$ with respect to the vehicle treated group or naïve group respectively. Panel (I,J) shows data from regression analysis for GILZ and TLR4 transcripts in mice administered saline (I) or LPS (J).

preferably bind high-affinity MR and mediate neuroprotective responses. High GC on the other hand promote neuronal injury by increasing inflammation and potentiating other cytotoxic mechanisms (Duque Ede and Munhoz, 2016; Kalafatakis et al., 2016). We postulated that since GILZ is an early and rapidly induced gene by GC, it is likely that it influences these processes.
Intraperitoneal LPS upregulate inflammatory cytokines which in turn increase glucocorticoid secretion. Yet, considerable evidence suggest that the LPS induced neuroinflammation is aggravated by higher circulating GC or chronic stress induced GC or exogenous GC due to development of glucocorticoid resistance and impaired feedback (Weidenfeld and Yirmiya, 1996; Pérez-Nievas et al., 2010; Vyas et al., 2016). We 
observed that the GILZ expression was reduced in the hippocampus in this model correlating negatively with the increased inflammatory markers. Others have reported reduced GILZ expression in LPS induced uveitis (Gu et al., 2017). Previously, reduced GILZ expression and elevated inflammatory cytokines in circulating lymphocytes were correlated with lower hippocampal volume in patients with major depressive disorder (Frodl et al., 2012). In contrast, water immersion stress that enhance endogenous GC was shown to upregulate neuronal GILZ in the mouse hippocampus (Yachi et al., 2007). The discrepancy could be attributed to the nature of the stimuli and the method used, in situ hybridization vs. quantitative PCR (this study). Pertinently, GILZ has been suggested to exert cell-context dependent functions ranging from inhibition of inflammation and immune responses to modulation of proliferation and cell death in multiple cell types (Cohen et al., 2006; Liu et al., 2013; Pan et al., 2014).

Data from serial gene expression analysis that characterized the global transcriptional effects of MR and GR signaling in GR deficient HT-22 neuronal cells showed that the GILZ is upregulated only in cells co-expressing MR and GR (Rozeboom, 2008). These observations suggest that the GILZ could contribute to the process of neuronal survival and apoptosis, perhaps in a GC concentration dependent manner. Our observations of higher GILZ expression in the brain of naïve mice suggest that in health GILZ is potentially upregulated by MR mediated transactivation. The reduced GILZ expression in LPS induced TLR-4 mediated inflammation perhaps reflects cytoplasmic GR

\section{REFERENCES}

Abrahám, I. M., Meerlo, P., and Luiten, P. G. (2006). Concentration dependent actions of glucocorticoids on neuronal viability and survival. Dose Response 4, 38-54. doi: 10.2203/dose-response.004.01.004. abraham

Ayroldi, E., and Riccardi, C. (2009). Glucocorticoid-induced leucine zipper (GILZ): a new important mediator of glucocorticoid action. FASEB J. 23, 3649-3658. doi: 10.1096/fj.09-134684

Ayyar, V. S., Almon, R. R., Jusko, W. J., and DuBois, D. C. (2015). Quantitative tissue-specific dynamics of in vivo GILZ mRNA expression and regulation by endogenous and exogenous glucocorticoids. Physiol. Rep. 3:e12382. doi: 10.14814/phy2.12382

Bergann, T., Fromm, A., Borden, S. A., Fromm, M., and Schulzke, J. D. (2011). Glucocorticoid receptor is indispensable for physiological responses to aldosterone in epithelial $\mathrm{Na}^{+}$channel induction via the mineralocorticoid receptor in a human colonic cell line. Eur. J. Cell Biol. 90, 432-439. doi: 10.1016/j.ejcb.2011.01.001

Berrebi, D., Bruscoli, S., Cohen, N., Foussat, A., Migliorati, G., Bouchet-Delbos, L., et al. (2003). Synthesis of glucocorticoid-induced leucine zipper (GILZ) by macrophages: an anti-inflammatory and immunosuppressive mechanism shared by glucocorticoids and IL-10. Blood 101, 729-738. doi: 10.1182/blood2002-02-0538

Catorce, M. N., and Gevorkian, G. (2016). LPS-induced murine neuroinflammation model: main features and suitability for pre-clinical assessment of nutraceuticals. Curr. Neuropharmacol. 14, 155-164. doi: 10.2174/1570159x14666151204122017

Cohen, N., Mouly, E., Hamdi, H., Maillot, M. C., Pallardy, M., Godot, V., et al. (2006). GILZ expression in human dendritic cells redirects their maturation and prevents antigen-specific T lymphocyte response. Blood 107, 2037-2044. doi: 10.1182/blood-2005-07-2760 retention with consequent reduced GILZ transactivation. Similar inverse correlation between the TLR-4 and GILZ expression is reported in alveolar macrophages (Hoppstadter et al., 2012).

Taken together, our data suggest a novel role for GILZ as a modulator of the neuroprotective and neuroinflammatory responses of GC. Further studies are underway to evaluate the effects of stress and inflammation in GILZ overexpressing mice. In addition, since GILZ sequesters activated NF-кB p65 in the cytoplasm and prevent inflammatory responses, it may represent a viable candidate for drug development (Ayroldi and Riccardi, 2009). Indeed, we have recently reported that analogs of the p65 binding motif of GILZ can inhibit amyloid beta induced inflammatory responses in mixed brain cells (Srinivasan et al., 2016).

\section{AUTHOR CONTRIBUTIONS}

MS, DH and DL were involved in concept development, data interpretation and manuscript preparation. EW and $\mathrm{DH}$ performed the animal experiments, data acquisition and interpretation.

\section{FUNDING}

This work was supported by the NIH (1R41 AG053117) to MS, from Alzheimer's Association (IIRG-11-206418) and the National Institute on Aging, NIH (5R21AG042804 and 1R21AG047) to DL.

D’Adamio, F., Zollo, O., Moraca, R., Ayroldi, E., Bruscoli, S., Bartoli, A., et al (1997). A new dexamethasone-induced gene of the leucine zipper family protects $\mathrm{T}$ lymphocytes from TCR/CD3-activated cell death. Immunity 7 , 803-812. doi: 10.1016/s1074-7613(00)80398-2

Duque Ede, A., and Munhoz, C. D. (2016). The pro-inflammatory effects of glucocorticoids in the brain. Front. Endocrinol. Lausanne. 7:78. doi: 10.3389/fendo.2016.00078

Frank, M. G., Miguel, Z. D., Watkins, L. R., and Maier, S. F. (2010). Prior exposure to glucocorticoids sensitizes the neuroinflammatory and peripheral inflammatory responses to E. coli lipopolysaccharide. Brain Behav. Immun. 24, 19-30. doi: 10.1016/j.bbi.2009.07.008

Frodl, T., Carballedo, A., Hughes, M. M., Saleh, K., Fagan, A., Skokauskas, N., et al. (2012). Reduced expression of glucocorticoid-inducible genes GILZ and SGK-1: high IL-6 levels are associated with reduced hippocampal volumes in major depressive disorder. Transl. Psychiatry 2:e88. doi: 10.1038/tp.2012.14

Gu, R., Lei, B., Shu, Q., Li, G., and Xu, G. (2017). Glucocorticoid-induced leucine zipper overexpression inhibits lipopolysaccharide-induced retinal inflammation in rats. Exp. Eye Res. 165, 151-163. doi: 10.1016/j.exer.2017.02. 011

Hoppstadter, J., Diesel, B., Eifler, L. K., Schmid, T., Brüne, B., and Kiemer, A. K. (2012). Glucocorticoid-induced leucine zipper is downregulated in human alveolar macrophages upon toll-like receptor activation. Eur. J. Immunol. 42, 1282-1293. doi: 10.1002/eji.201142081

Huang, C. W., Lui, C. C., Chang, W. N., Lu, C. H., Wang, Y. L., and Chang, C. C. (2009). Elevated basal cortisol level predicts lower hippocampal volume and cognitive decline in Alzheimer's disease. J. Clin. Neurosci. 16, 1283-1286. doi: 10.1016/j.jocn.2008.12.026

Ifuku, M., Katafuchi, T., Mawatari, S., Noda, M., Miake, K., Sugiyama, M., et al. (2012). Anti-inflammatory/anti-amyloidogenic effects of plasmalogens in lipopolysaccharide-induced neuroinflammation in adult mice. J. Neuroinflammation 9:197. doi: 10.1186/1742-2094-9-197 
Kalafatakis, K., Russell, G. M., Zarros, A., and Lightman, S. L. (2016). Temporal control of glucocorticoid neurodynamics and its relevance for brain homeostasis, neuropathology and glucocorticoid-based therapeutics. Neurosci. Biobehav. Rev. 61, 12-25. doi: 10.1016/j.neubiorev.2015.11.009

Liu, J., Zhang, M., Niu, C., Luo, Z., Dai, J., Wang, L., et al. (2013). Dexamethasone inhibits repair of human airway epithelial cells mediated by glucocorticoidinduced leucine zipper (GILZ). PLoS One 8:e60705. doi: 10.1371/journal.pone. 0060705

Lupien, S. J., Nair, N. P., Briere, S., Maheu, F., Tu, M. T., Lemay, M., et al. (1999). Increased cortisol levels and impaired cognition in human aging: implication for depression and dementia in later life. Rev. Neurosci. 10, 117-139. doi: 10.1515/revneuro.1999.10.2.117

Lykhmus, O., Mishra, N., Koval, L., Kalashnyk, O., Gergalova, G., Uspenska, K., et al. (2016). Molecular mechanisms regulating LPS-induced inflammation in the brain. Front. Mol. Neurosci. 9:19. doi: 10.3389/fnmol.2016.00019

Mazzon, E., Bruscoli, S., Galuppo, M., Biagioli, M., Sorcini, D., Bereshchenko, O., et al. (2014). Glucocorticoid-induced leucine zipper (GILZ) controls inflammation and tissue damage after spinal cord injury. CNS Neurosci. Ther. 20, 973-981. doi: 10.1111/cns.12315

Nazem, A., Sankowski, R., Bacher, M., and Al-Abed, Y. (2015). Rodent models of neuroinflammation for Alzheimer's disease. J. Neuroinflammation 12:74. doi: 10.1186/s12974-015-0291-y

Newton, R. (2000). Molecular mechanisms of glucocorticoid action: what is important? Thorax 55, 603-613. doi: 10.1136/thorax.55.7.603

Ogita, K., Sugiyama, C., Acosta, G. B., Kuramoto, N., Shuto, M., Yoneyama, M., et al. (2012). Opposing roles of glucocorticoid receptor and mineralocorticoid receptor in trimethyltin-induced cytotoxicity in the mouse hippocampus. Neurosci. Lett. 511, 116-119. doi: 10.1016/j.neulet.2012.01.052

Pan, G., Cao, J., Yang, N., Ding, K., Fan, C., Xiong, W. C., et al. (2014). Role of glucocorticoid-induced leucine zipper (GILZ) in bone acquisition. J. Biol. Chem. 289, 19373-19382. doi: 10.1074/jbc.M113.535237

Pérez-Nievas, B. G., Madrigal, J. L., García-Bueno, B., Zoppi, S., and Leza, J. C. (2010). Corticosterone basal levels and vulnerability to LPS-induced neuroinflammation in the rat brain. Brain Res. 1315, 159-168. doi: 10.1016/j. brainres.2009.12.014

Qin, L., Wu, X., Block, M. L., Liu, Y., Breese, G. R., Hong, J. S., et al. (2007). Systemic LPS causes chronic neuroinflammation and progressive neurodegeneration. Glia 55, 453-462. doi: 10.1002/glia.20467

Ray, B., Bailey, J. A., Sarkar, S., and Lahiri, D. K. (2009). Molecular and immunocytochemical characterization of primary neuronal cultures from adult rat brain: differential expression of neuronal and glial protein markers. J. Neurosci. Methods 184, 294-302. doi: 10.1016/j.jneumeth.2009.08.018

Rozeboom, A. M. (2008). Functions of the Mineralocorticoid Receptor in the Hippocampus. PhD, Cellular and Molecular Biology. Ann Arbor, MI: The University of Michigan.
Rupprecht, R., Reul, J. M., van Steensel, B., Spengler, D., Söder, M., Berning, B., et al. (1993). Pharmacological and functional characterization of human mineralocorticoid and glucocorticoid receptor ligands. Eur. J. Pharmacol. 247, 145-154. doi: 10.1016/0922-4106(93)90072-h

Shi, X., Shi, W., Li, Q., Song, B., Wan, M., Bai, S., et al. (2003). A glucocorticoidinduced leucine-zipper protein, GILZ, inhibits adipogenesis of mesenchymal cells. EMBO Rep. 4, 374-380. doi: 10.1038/sj.embor.embor805

Srinivasan, M., Bayon, B., Chopra, N., and Lahiri, D. K. (2016). Novel nuclear factor- $\mathrm{B}$ targeting peptide suppresses $\beta$-amyloid induced inflammatory and apoptotic responses in neuronal cells. PLoS One 11:e0160314. doi: 10.1371/journal.pone.0160314

Srinivasan, M., and Janardhanam, S. (2011). Novel p65 binding glucocorticoidinduced leucine zipper peptide suppresses experimental autoimmune encephalomyelitis. J. Biol. Chem. 286, 44799-44810. doi: 10.1074/jbc.M111. 279257

Srinivasan, M., and Lahiri, D. K. (2017). Glucocorticoid-induced leucine zipper in central nervous system health and disease. Mol. Neurobiol. 54, 8063-8070. doi: 10.1007/s12035-016-0277-5

Vyas, S., Rodrigues, A. J., Silva, J. M., Tronche, F., Almeida, O. F., Sousa, N., et al. (2016). Chronic stress and glucocorticoids: from neuronal plasticity to neurodegeneration. Neural Plast. 2016:6391686. doi: 10.1155/2016/63 91686

Weidenfeld, J., and Yirmiya, R. (1996). Effects of bacterial endotoxin on the glucocorticoid feedback regulation of adrenocortical response to stress. Neuroimmunomodulation 3, 352-357. doi: 10.1159/000097295

Yachi, K., Inoue, K., Tanaka, H., Yoshikawa, H., and Tohyama, M. (2007). Localization of glucocorticoid-induced leucine zipper (GILZ) expressing neurons in the central nervous system and its relationship to the stress response. Brain Res. 1159, 141-147. doi: 10.1016/j.brainres.2007.05.024

Yu, Z., Tang, L., Chen, L., Li, J., Wu, W., and Hu, C. (2015). Capillarisin suppresses lipopolysaccharide-induced inflammatory mediators in BV2 microglial cells by suppressing TLR4-mediated NF- $\mathrm{B}$ and MAPKs signaling pathway. Neurochem. Res. 40, 1095-1101. doi: 10.1007/s11064-015-1567-4

Conflict of Interest Statement: The authors declare that the research was conducted in the absence of any commercial or financial relationships that could be construed as a potential conflict of interest.

Copyright (c) 2019 Witek, Hickman, Lahiri and Srinivasan. This is an open-access article distributed under the terms of the Creative Commons Attribution License (CC BY). The use, distribution or reproduction in other forums is permitted, provided the original author(s) and the copyright owner(s) are credited and that the original publication in this journal is cited, in accordance with accepted academic practice. No use, distribution or reproduction is permitted which does not comply with these terms. 